Tropical Journal of Pharmaceutical Research December 2010; 9 (6): 525-531

(C) Pharmacotherapy Group,

Faculty of Pharmacy, University of Benin,

Benin City, 300001 Nigeria.

All rights reserved.

Research Article

Available online at http://www.tjpr.org

\title{
The Effect of Polymer Molecular Weight on Citrate Crosslinked Chitosan Films for Site-Specific Delivery of a Non-Polar Drug
}

\section{Soheyla Honary*, Behnam Hoseinzadeh and Payman Shalchian \\ Mazandaran University of Medical Sciences, School of Pharmacy, Pharmaceutical Sciences Research Center, Sari, Iran}

\begin{abstract}
Purpose: To develop citrate crosslinked chitosan films using chitosan of different molecular weights (MW) in order to achieve site-specific delivery of a model non-polar drug, indomethacin.

Methods: Films prepared with different molecular weights of chitosan and incorporating indomethacin as a non-polar model drug were obtained by a casting/solvent evaporation method. The chitosan films were crosslinked by dipping in varying concentrations of sodium citrate solution and for different crosslinking times. The films were assessed by, amongst others, scanning electron microscopy (SEM), dissolution studies and differential scanning calorimetry (DSC) for surface morphology, drug release and ingredient compatibility, respectively.

Results: Crosslinking time and concentration of crosslinking agent significantly $(p<0.05)$ influenced the in vitro release of indomethacin as well as swelling of the films. Also, the higher the molecular weight $(M W)$ of chitosan the lower the drug release rate $(p<0.05)$. Furthermore, film swelling index rose as chitosan MW decreased ( $p<0.05$ ). The practical absence of the sharp endothermic peak characteristic of indomethacin in the films suggests that crosslinking may have transformed the drug from the crystalline to the amorphous state.

Conclusion: The citrate-crosslinked chitosan films can be modulated to vary swelling and drug release at pH 3.5 and 6.2; this feature makes them useful tools for designing site-specific delivery systems.
\end{abstract}

Keywords: Chitosan film, Sodium citrate, Site-specific delivery, Crosslinking, Indomethacin 


\section{INTRODUCTION}

A major feature of medicines development today is the design of suitable vehicles for drug delivery. Delivery vehicles for therapeutic compounds must be biocompatible and nontoxic [1]. Many of these systems are $\mathrm{pH} /$ thermo-sensitive or osmotically driven [2]. Thin polymer films have also been employed to control drug release for various routes of administration, such as transdermal, colonic, localized, and ophthalmic. Mucoadhesive polymeric film formulations can circumvent limitations caused by conventional vaginal formulations such as tablets and creams [3]. For example, the release of paclitaxel from PVP-g-PLGA film was found to be continuous and not preceded by initial drug burst [4].

Time- or pH-dependent polymers are often used to target delivery of therapeutic substances to sites of action [5]. Several studies have associated drug release kinetics with the type of drug and device geometry. For instance, Klose et al studied these factors in PLGA-based drug delivery systems [6] while results from other studies indicate that polymer films are promising carriers for ophthalmic, localized and transdermal drug delivery systems [7]. Various polymethacrylate films have been evaluated for insulin colonic drug delivery [8].

Chitosan [a (1-4) 2-amino-2-deoxy-beta-DGlucan] is the deacetylated derivative of chitin. It has recently attracted much interest in the biomedical industry because of its biodegradability, biocompatibility, antimicrobial activity, and accelerated wound healing properties [9]. Due to its unique polymeric cationic character as well as gel and film forming properties, chitosan has been extensively examined for various pharmaceutical applications. Several drug delivery formulations based on chitosan, such as film, beads, microspheres, etc, have been prepared using chemical cross-linking methods. For example, Emmanuel et al demonstrated the in vitro and in vivo zeroorder drug release properties and biocompatibility of a chitosan-based implantable film and its potential usefulness in the sustained and local delivery of antineoplastic agents such as paclitaxel [10].

When chitosan is dissolved in dilute acetic acid solutions, the amino groups become protonated and associated with acetate counter-ions, making the charged polymer soluble [11]. Chitosan films have been usually prepared by chemical crosslinking via electrostatic interaction between multivalent phosphates and chitosan in the formulation [12]. Chitosan crosslinked films swell under acidic conditions due to the ionization of amino groups but remains in a shrunken state under neutral conditions. Chitosan itself has intragastric-floating characteristics and can cause prolonged retention of the dosage form in the stomach [13].

Controlled release of ciprofloxacin hydrochloride from chitosan/polyethylene glycol blend films produced by the castingsolvent evaporation method has been evaluated [14]. It has also been shown that due to electrostatic interaction between sodium citrate and chitosan, citrate/chitosan film may be useful in drug delivery such as site-specific controlled release in stomach and also as epidermal sheets for humans, rats, and rabbits [15]. Polymer molecular weight has a major effect on chitosan properties [16], and it has also been shown that the thermo-sensitive characteristics, appearance and structure of the hydrogel as well as drug release from it are affected by chitosan MW and degree of deacetylation [17]. In this paper, we sought to prepare citrate-crosslinked chitosan films using three different molecular weights (MWs) of chitosan and investigated whether or not chitosan MW affects some properties of the indomethacincontaining films. 


\section{EXPERIMENTAL}

\section{Materials}

Indomethacin (1-(p-chlorobenzoyl)-5-metho$x y-2$-methylindole-3-acetic acid), produced by Medichem, Spain, was used as a model hydrophobic drug. Low, medium and high molecular weight grades of chitosan (85\% deacetylation) were purchased from Fluka AG, Switzerland while sodium citrate (analytical grade) and other reagents were purchased locally and used as received.

\section{Turbidimetric titration}

The interaction between sodium citrate and chitosan was investigated by turbidimetric titration [18]. Briefly, a solution of $0.2 \mathrm{~g} / \mathrm{L}$ sodium citrate and $0.2 \mathrm{~g} / \mathrm{L}$ chitosan at $\mathrm{pH} 1.0$ was prepared. The titrant $(0.2 \mathrm{M} \mathrm{NaOH})$ was delivered with a microburette into $50 \mathrm{ml}$ of the citrate/chitosan solution with constant stirring at $25 \pm 0.5^{\circ} \mathrm{C}$ and with the $\mathrm{pH}$ monitored by a digital pH meter (Metrohm 780, Switzerland). Changes in turbidity were monitored at 420 $\mathrm{nm}$ with an UV-Vis spectrophotometer (Genesys TM2, USA and reported as $100 \%$ transmittance.

\section{Preparation of crosslinked chitosan films}

Chitosan solution ( $4 \% \mathrm{w} / \mathrm{v})$, was prepared by dissolving chitosan in $4 \% \mathrm{w} / \mathrm{v}$ acetic acid and then indomethacin $(1 \% \mathrm{w} / \mathrm{v})$ was dispersed in the solution. This was done for each MW grade of chitosan. The mixture $(100 \mathrm{ml})$ was left to stand until trapped air bubbles disappeared and then was poured on a glass plate. The poured solution was allowed to dry in a hot air oven (Pars Azma 1597) at $37^{\circ} \mathrm{C}$ to constant weight. The resulting dry films were cut into $4 \mathrm{~cm}$ diameter disks and crosslinked soaking in $100 \mathrm{ml}$ aqueous solution of sodium citrate $4{ }^{\circ} \mathrm{C}$. Other crosslinking conditions were: $0.5-10 \% \mathrm{w} / \mathrm{v}$ sodium citrate; solution $\mathrm{pH}$ of $5-7$; and crosslinking time of $0.5-4.0 \mathrm{~h}$. The crosslinked films were then rinsed in $20 \mathrm{ml}$ of distilled water, transferred to a glass plate and oven-dried at $37^{\circ} \mathrm{C}$ to a constant weight. Drug loss during the crosslinking process was determined spectrophotometrically at $318 \mathrm{~nm}$.

\section{Measurement of film thickness}

The thickness of the dried films was measured with a micrometer (model 2050-08, Mitutoyo, Japan). Five measurements were taken at randomly selected points on each specimen and the mean thickness taken.

\section{Morphological studies}

The surface morphology of the crosslinked films was assessed by scanning electron microscopy (model 2360, Leo Oxford, England) after the films were first coated with gold.

\section{Evaluation of swelling ratio}

Blank citrate/chitosan films were suspended in glass bottles containing $250 \mathrm{ml}$ of medium (either phosphate buffer, $\mathrm{pH}$ 6.2, or acetate buffer, $\mathrm{pH}$ 3.5) and placed in a shaker waterbath (at $\left.37^{\circ} \mathrm{C}, 50 \mathrm{rpm}\right)$. At $30 \mathrm{~min}$ intervals, the films were taken out, the excess water blotted out carefully with filter paper from the film surface and then weighed immediately. The swelling ratio $\left(\mathrm{W}_{\mathrm{t}} / \mathrm{W}_{0}\right)$ was determined (where $W_{t}$ was the film weight at time $t$ and $W_{0}$ was the initial film weight).

\section{Release studies}

Indomethacin release from the films was evaluated by USP dissolution method V (paddle over disk method) in $750 \mathrm{ml}$ phosphate buffer ( $\mathrm{pH}$ 6.2) or acetate buffer $(\mathrm{pH} 3.5)$ at $37{ }^{\circ} \mathrm{C}$ and at a stirring rate of 50 rpm, using Erweka dissolution tester (model DT 80$)$. Samples $(5 \mathrm{ml})$ were withdrawn from the medium at various time intervals and the medium replenished immediately with the same volume of fresh dissolution medium. The amount of dissolved indomethacin in the sample taken was measured (after filtration) spectrophotometrically at $318 \mathrm{~nm}$. 
Indomethacin concentration in each sample was calculated according to Eq 1

Cn $=$ Cnmass $+\frac{V}{V t} \sum_{s=1}^{n-1}$ Cmass

where $\mathrm{Cn}=$ true concentration of sample,

Cnmass = apparent concentration of sample,

$\mathrm{V}=$ volume of sample, and $\mathrm{Vt}=$ total volume

of dissolution fluid.

\section{Differential scanning calorimetry (DSC)}

Differential scanning calorimetric studies were conducted in a Perkin Elmer DSC facility,using a sample size of approx. $5 \mathrm{mg}$ in a loosely covered aluminum pan and heated in nitrogen atmosphere from 50 to $250{ }^{\circ} \mathrm{C}$ at a rate of $10^{\circ} \mathrm{C} / \mathrm{min}$. An empty loosely covered aluminum pan was used as the reference.

\section{Statistical analysis}

Experimental results were expressed as mean \pm standard deviation (SD). Student's $t$ test and one-way analysis of variance (ANOVA) were applied to check significant differences in drug release, and swelling studies for different film formulations. Differences were considered to be statistically significant at $p<0.05$.

\section{RESULTS}

\section{Film thickness and drug content}

Table 1 shows the mean thickness and drug content of the films prepared at varying combinations of crosslinking concentration and time. The results show that there was no significant difference $(p>0.05)$ between the films in terms of film thickness or drug content.

\section{Citrate - chitosan interaction}

The turbidimetric titration curve of sodium citrate/chitosan (not shown) indicated that in acidic conditions ( $\mathrm{pH} 1$ - 4), the solution was clear; thereafter, it became turbid as the $\mathrm{pH}$ further rose but reverted to a clear solution (i.e., no interaction) at $\mathrm{pH} \geq 7$.
Table 1: Mean thickness and drug content of crosslinked chitosan films

\begin{tabular}{|c|c|c|c|c|}
\hline Film code & $\mathrm{C}_{\mathrm{t}}(\mathrm{h})$ & $\begin{array}{c}\mathrm{C}_{\mathrm{c}} \\
(\%)\end{array}$ & $\begin{array}{c}\text { Film } \\
\text { thicknes } \\
s(\mu \mathrm{m}, \\
n=5)\end{array}$ & $\begin{array}{c}\text { Drug } \\
\text { content } \\
(\%)\end{array}$ \\
\hline $\begin{array}{l}\text { Low MW } \\
\text { chitosan } \\
\text { F1 }\end{array}$ & - & - & $36.3 \pm 1.2$ & 100.0 \\
\hline $\mathrm{F} 2$ & 0.5 & 0.5 & $40.0 \pm 0.8$ & 100.0 \\
\hline F3 & 0.5 & 10 & $36.7 \pm 0.5$ & 98.5 \\
\hline F4 & 4.0 & 0.5 & $39.3 \pm 1.7$ & 98.5 \\
\hline F5 & 4.0 & 10 & $33.3 \pm 1.2$ & 98.6 \\
\hline $\begin{array}{l}\text { Medium M } \\
\text { chitosan } \\
\text { F6 } \\
\text { F7 }\end{array}$ & - & $\begin{array}{c}- \\
0.5\end{array}$ & $\begin{array}{l}38.3 \pm 1.7 \\
33.3 \pm 1.2\end{array}$ & $\begin{array}{l}100.3 \\
100.0\end{array}$ \\
\hline F8 & 0.5 & 10 & $43.3 \pm 1.2$ & 99.1 \\
\hline F9 & 4.0 & 0.5 & $41.7 \pm 1.7$ & 99.0 \\
\hline $\mathrm{F} 10$ & 4.0 & 10 & $34.7 \pm 1.7$ & 99.4 \\
\hline $\begin{array}{l}\text { High MW } \\
\text { chitosan } \\
\text { F11 } \\
\text { F12 }\end{array}$ & - & $\overline{0 .}$ & $\begin{array}{l}34.7 \pm 1.2 \\
35.0 \pm 1.6\end{array}$ & $\begin{array}{l}98.7 \\
99.3\end{array}$ \\
\hline $\mathrm{F} 13$ & 0.5 & 10 & $39.7 \pm 0.9$ & 98.4 \\
\hline $\mathrm{F} 14$ & 4.0 & 0.5 & $37.0 \pm 1.4$ & 98.1 \\
\hline F15 & 4.0 & 10 & $38.3 \pm 0.9$ & 98.6 \\
\hline
\end{tabular}

\section{Film appearance}

SEM photomicrographs (not shown) indicate that the inner surface of the films (which interfaced with the glass plate) was smooth while the outer surface was relatively rough. Crosslinking hardly had any noticeable effect on the surface morphology of the films. However, all the films were yellowish cream in colour, with the colour deepening and film texture becoming more tender with increase in crosslinking time.

\section{Swelling ratio}

The swelling data are shown in Fig 1. They indicate that at $\mathrm{pH}$ 6.2, film swelling for all three chitosan MW grades was significantly $(p<0.05)$ affected by crosslinking time. All the MW grades showed higher swelling in acid conditions ( $\mathrm{pH}$ 3.5) than at $\mathrm{pH}$ 6.2. Furthermore, at $\mathrm{pH}$ of 3.5, swelling decreased as MW of chitosan rose. 


\section{Drug release studies:}

Fig 2 shows that indomethacin was released rapidly from the film matrix at $\mathrm{pH} 3.5$. Drug release was independent of either crosslinking time or concentration $(p>0.05)$. The results also show that drug release rate decreased significantly at $\mathrm{pH} 3.5(p<0.05)$ as chitosan MW increased. On the other hand, at $\mathrm{pH}$ 6.2, as Fig 3 indicates, drug release was significantly decreased by cross linking time and/or concentration of crosslinking agent $(p<0.05)$. The greater the crosslinking time, the lower was the drug release rate

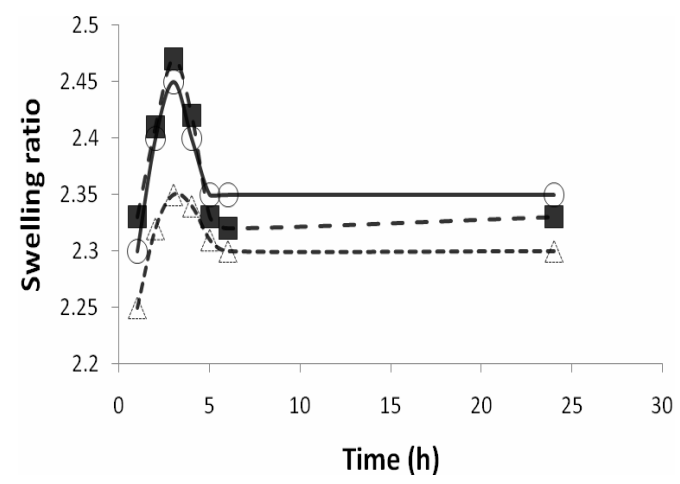

Fig 1: Swelling ratio of chitosan films $(\circ=L M W$, - = MMW, $\Delta=\mathrm{HMW}$ ) in $\mathrm{pH} 3.5$ medium

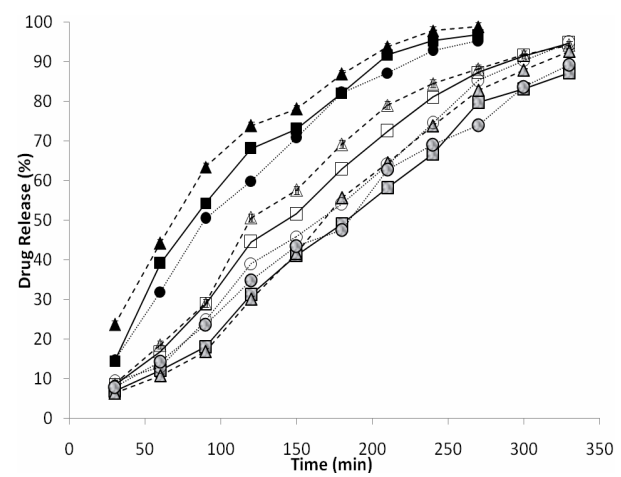

Fig 2: Release of indomethacin from chitosan films in pH 3.5 medium: $\square=F 1 ; \square=F 3$; $\quad=F 5$; $\Delta=\mathrm{F} 6, \quad \Delta \mathrm{F} 8, \quad \Delta \mathrm{F} 10, \bullet \mathrm{F} 11, \circ \mathrm{F} 13, \bullet$ F15 (See Table 1 code key)

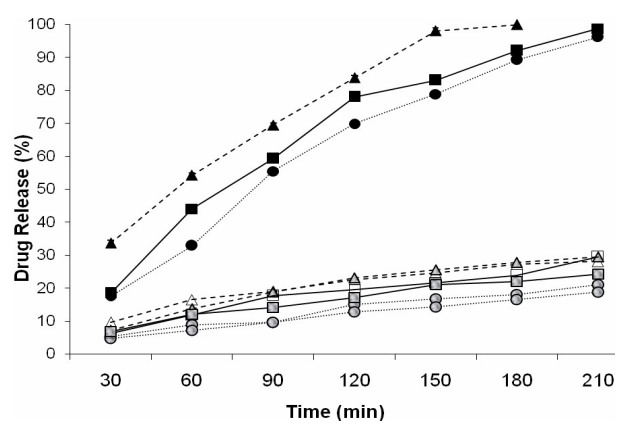

Fig 3: Release of indomethacin from chitosan films in $\mathrm{pH} 6.2$ medium $(\boldsymbol{\square}=\mathrm{F} 1 ; \square=\mathrm{F} 3$; $\mathbf{\square}=\mathrm{F} 5$; $\boldsymbol{\Delta}=\mathrm{F} 6 ; \Delta=\mathrm{F} 8 ; \boldsymbol{\Delta}=\mathrm{F} 10 ; \bullet=\mathrm{F} 11 ; \circ=\mathrm{F} 13 ; \bullet=$ F15 (Code key as in Table 1)

There was no significant difference between low and medium MW grades of chitosan in terms of the drug release rate of the films $(p>$ 0.05). However, high MW chitosan film showed a slight decrease in the rate of drug release in acid conditions.

\section{Thermal characteristics}

The DSC thermograms of pure indomethacin and some of the chitosan films are shown in Fig 4. The thermogram of.indomethacin (A) showed a sharp endothermic peak at $165^{\circ} \mathrm{C}$ which indicates the melting transition of the drug. The thermogram of $\mathrm{F} 6(\mathrm{~B})$, which was similar to those of F1, F5, F10, F11 and F15 (not shown), manifested a broad endothermic peak. A combination of the highest crosslinking concentration (10\% sodium citrate) and longest cross-linking time (4 h) for the different $\mathrm{MW}$ grades of chitosan caused a depression of the endothermic peak in the film thermograms by approximately 57 , 12 and $5{ }^{\circ} \mathrm{C}$ for $\mathrm{F} 1, \mathrm{~F} 6$ and $\mathrm{F} 11$, respectively.

\section{DISCUSSION}

Citrate is an anion with three carboxylic groups and chitosan is polybasic with cations. The charge densities of citrate and chitosan are mainly controlled by solution $\mathrm{pH} 13-14]$. 
Under neutral and weakly acidic conditions, the degree of ionization of sodium citrate significantly decreased owing to the weak acid characteristics of citric acid. In contrast, chitosan, being a weak polybase, showed a marked decrease in the ionization of its amine groups when solution $\mathrm{pH}$ increased to over 6.0 (chitosan pKa =6.3)[19].

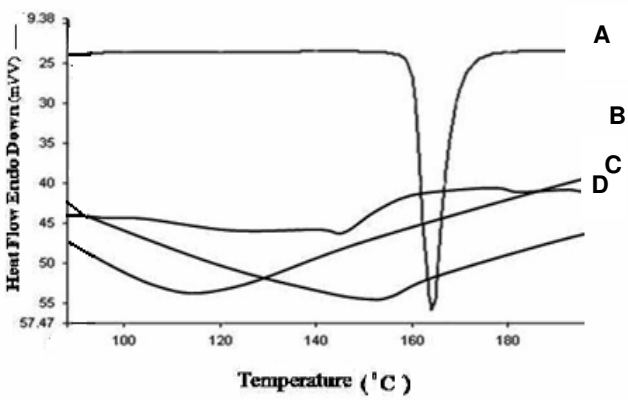

Fig 4: DSC thermograms of pure indomethacin $(A)$ and chitosan films $(B=F 6, C=F 11$, and $D=F 1)$ (Code key as in Table 1)

\section{Film swelling}

The $\mathrm{pH}$ of the medium had a major effect on chitosan film swelling due to the ionization of both the sodium citrate and chitosan. Swelling ratio was lowest at $\mathrm{pH}$ of 5.5 and 6.5 , owing to electrostatic attraction between citrate and chitosan. Reduction in $\mathrm{pH}$ weakened the salt bonds and, therefore, facilitated chitosan film swelling. However, increase in $\mathrm{pH}$ above 6.5 would probably also have weakened the salt-bonds and produced a higher swelling ratio.

\section{Drug release}

Drug release from the films was sensitive to $\mathrm{pH}$ because electrostatic interaction between the citrate anion and chitosan amine groups were influenced by solution $\mathrm{pH}$. Decrease in $\mathrm{pH}$ weakened the salt bonds and therefore, facilitated film swelling, thereby making it more porous and accelerating drug release. $\mathrm{pH}$ also has been reported to have a slight effect on the solubility of indomethacin [20]. A lower $\mathrm{pH}$ leads to improved solubility of drug and this in turn results in higher drug release rate. However, compared to the strong influence of $\mathrm{pH}$ on the film matrix, $\mathrm{pH}$ effects on drug solubility was negligible. At low $\mathrm{pH}$ (1.0 - 3.5), sodium citrate and chitosan would be in a dissociated state and hence drug release from the films was rapid. As the results show the greater the crosslinking time, the lower was the drug release rate, This may be attributed to a higher degree of crosslinking in the matrix, leading to delay in the diffusional release of the drug. A slight decrease in the rate of drug release in acid conditions for high MW chitosan film maybe related to its higher viscosity compared with low and medium MWs.

Although the protonation constant (pKa) of chitosan slightly decreases (from 6.51 to 6.39) with decrease in its MW [19], in acid conditions ( $\mathrm{pH}$ 3.5), a slight decrease in $\mathrm{pKa}$ appeared not to have played a major role in drug release rate due to high dissociation at all chitosan MWs.

\section{Thermal characteristics}

The absence of a sharp endothermic melting peak for indomethacin in the thermograms of these films may be due to the conversion of the incorporated indomethacin from the crystalline to the more soluble amorphous state.

\section{CONCLUSION}

The variations in citrate-crosslinked chitosan film swelling and as well as in drug release rate in medium $\mathrm{pH}$ of 3.5 and 6.2 suggest that this type of films can be modulated to achieve site-specific delivery in the stomach.

\section{ACKNOWLEDGEMENT}

This work was supported financially by Mazandaran University of Medical Sciences, Sari, Iran. 


\section{REFERENCES}

1. Tsukagoshi T, Kondo Y, Yoshino N. Preparation of thin polymer films with controlled drug release. Colloids Surfaces B 2007; 57: 219-225.

2. Guo BL, Yuan JF, Gan QY. Preparation and characterization of temperature and $\mathrm{pH}$ sensetive chitosan material and its controlled release on coenzyme $A$. Colloids Surfaces $B$ 2007; 58: 151-156.

3. Yoo JW, Deharmala $K$, Lee $\mathrm{CH}$. The physicodynamic properties of mucoadhesive polymeric films developed as female controlled drug delivery system. Int J Pharm 2006; 309: 139-145.

4. Westedt $U$, Wittmar $M$, Hellwing $M$, Hanefeld $P$, Greiner A, Schaper AK, Kissel T. Paclitaxel releasing films consisting of poly(vinyl alcohol)graft-poly(lactide-co-glycolide) and their potential as biodegradable stent coatings. $J$ Control Release 2006; 11: 235-246.

5. Tuovinen L, Peltonen S, Liikola M, Hotakainen M, Lahtela-Kaaaonen $M$, Poso A, Jarvinen $K$. Drug release from starch-acetate microparticles and films with and without incorporated $\alpha$ - amylase. Biomaterials 2004; 25: 4355-4362.

6. Klose D, Siepmann F, Elkharraz K, Siepmann J. PLGA-based drug delivery system: Importance of the type of drug and device geometry. Int $J$ Pharm 2008; 354: 95-103.

7. Wang Y, Challa P, Epstein DL, Yuan F. Controlled release of ethacrynic acid from poly(lactide-coglycolide) films for glaucoma treatment. Biomaterials 2004; 25: 4279-4285.

8. Padula $C$, Nicoli $S$, Colombo $P$, Santi $P$. Singlelayer trnsdermal film containing lidocaine: Modulation of drug release. Eur $J$ Pharm Biopharm 2008; 66: 422-428.

9. Akhgari $A$, Farahmand $F$, Afrasiabi $H$, Sadeghi $F$, Vandamme TF. Permeability and swelling studies on free films containing insulin in combination with different polymethacrylates aimed for colonic drug delivery. Eur J pharm sci 2006; 28: 307-314.

10. Muzzarelli RAA. Chitins and chitosans for the repair of wounded skin, nerve, cartilage and bone. Carbohydrate Polymers 2009; 76: 167-182.
11. Ho EA, Vassileva V, Allen C, Piquette-Miller M. In vitro and in vivo characterization of novel biocompatible polymer-lipid implant system for the sustained delivery of paclitaxel. J Control Release. 2005; 104: 181-191.

12. Chen S, Liu M, Jin S, Wang B. Preparation of ioniccrosslinked chitosan-based gel beads and effect of reaction conditions on drug release behaviors. Intl J Pharm 2008; 349: 180-187.

13. Shu $X Z$, Zhu KJ. The influence of multivalent phosphate structure on the properties of ionically cross-linked chitosan films for controlled drug release. Eur $J$ Pharm Biopharm 2002; 54: 235-243.

14. Shu $X Z$, Zhu KJ. A novel approach to prepare tripolyphosphate/chitosan complex beads for controlled release drug delivery. Int $J$ Pharm 2000; 201: 51-58.

15. Wang Q, Dong Z, Du Y and Kennedy J. Controlled release of ciprofloxacin hydrochloride from chitosan/polyethylene glycol blend films. Carbohyd Polym 2007; 69: 336-343.

16. Rana V, Babita K, Goyal D and Tiwary A. Sodium citrate cross-linked chitosan films: optimization as substitude for human/rat/rabbit epidermal sheets. J Pharm Pharm Sci 2005; 8: 10-17.

17. Zhou HY, Chen XG, Kong M, Liu CS, Cha DS, Kennedy JF. Effect of molecular weight and degree of chitosan deacetylation on the preparation and characteristics of chitosan thermosensitive hydrogel as a delivery system. Carbohyd Polym 2008; 73: 265-273.

18. Shu $X Z$, Zhu KJ, Song $W$. Novel $p H$-sensitive citrate cross-linked chitosan film for drug controlled release. Int J Pharm 2001; 212: 19-28.

19. Wang QZ, Chen XG, Liu N, Wang SX, Liu CS, Meng $X H$, Liu CG. Protonation constants of chitosan with different molecular weight and degree of deacetylation. Carbohyd Polym 2006; 65: 194-201.

20. Nokhodchi A, Javadzadeh $Y$, Siahi M, Barzegar Jalali $M$. The effect of type and concentration of vehicles on the dissolution rate of a poorly soluble drug (indomethacin) from liquisolid compact. J Pharm Pharmaceut Sci 2005; 8: 18-28, 